\title{
Optical positions of radio emitting $\operatorname{stars}^{\star, \star \star}$
}

\author{
M. Assafin ${ }^{1,2}$, A.H. Andrei ${ }^{1}$, S.P. Puliaev ${ }^{1,3}$, E.G. Jilinski ${ }^{1,3}$, R. Vieira Martins ${ }^{1}$, G.G. Vieira ${ }^{2}$ and W.M. de \\ Oliveira $^{1,2}$ \\ 1 Observatório Nacional/CNPq, Brazil \\ 2 Observatório do Valongo/UFRJ, Brazil \\ 3 Pulkovo Observatory, Russia
}

Received August 9; accepted November 20, 1995

\begin{abstract}
In order to contribute to the alignment of the radio and optical reference frames, 50 stars with confirmed radio emission and published radio positions were observed by astrographical means. Additionally, two confirmed radio-stars of fainter magnitude were observed using a CCD direct camera with a long focus, large aperture telescope. The reductions are made relative to four catalogues: the Carlsberg Meridian Catalogue \#4, the International Reference Stars Catalogue, the Positions and Proper Motion Catalogue and the Astrographical Catalogue of Reference Stars. The best results were obtained with the CAMC catalogue. The plate error of a radio star position is 0 .'07 for both right ascension and declination, rising to $0.10-00^{\prime \prime} 20$ for bright stars. Ten of the program stars are not in the HIPPARCOS Input Catalogue and 12 do not belong to any of the major reference catalogues used. As soon as the HIPPARCOS results become available, its reference stars already measured in the plates will enable us to get positions for those 10 radio stars in the HIPPARCOS system.
\end{abstract}

Key words: astrometry — radio continuum: stars — reference systems

\section{Introduction}

The comparison between the fundamental optical reference frame and the radio reference frame, referred to extragalactic point sources by means of radio stars, is difficult because of the small number of stars exhibiting radio emission at the level of a few milliJanskys. Additional complications are brought about by variability and by the imperfect coincidence of the optical and radio regions of emission (Andrei et al. 1995). Positions of radio stars in the reference frame of well studied catalogues are useful to prevent future rotations of the HIPPARCOS system, due to the limit on proper motion accuracy $\left(\sim 00^{\prime \prime} 002 / \mathrm{yr}\right)$.

In this work, we concentrate on the astrographical determination of position for radio stars which have already published astrometric radio positions. The observations were made by the $0.40 \mathrm{~m}$ Zeiss Astrograph (focal distance $=2 \mathrm{~m}$ or $103 \mathrm{\prime \prime} / \mathrm{mm}$ plate scale) of the Universi-

Send offprint requests to: M. Assafin, Observatório Nacional/CNPq, R. Gal. José Cristino 77, CEP20921-400, Rio de Janeiro, Brazil

E-mail: massaf@on.br

*Based on observations made at Observatório Municipal de Campinas - SP and at Laboratório Nacional de Astrofísica/CNPq/MCT

${ }^{\star \star}$ Tables 2 and 4 also available in electronic form at CDS via ftp 130.79.128.5 dade Federal do Rio de Janeiro, in Campinas, São Paulo, $\left(\phi=-22^{\circ} 53^{\prime} 59^{\prime \prime} ; \lambda=3^{\mathrm{h}} 7^{\mathrm{m}} 19^{\mathrm{s}}\right)$. All measurements have been performed by the automatized PDS 1010A of the Observatório Nacional. Four catalogues were used for the plate reductions: the Carlsberg Meridian Catalogue - CAMC \#4 (1990), the Positions and Proper Motions PPM (Röser \& Bastian 1993), the International Reference Stars Catalogue - IRS (Corbin 1991) and the Astrographical Catalogue of Reference Stars - ACRS (Corbin \& Urban 1991). The average differences between catalogues match well the different results from the plates, thus providing a check on the reduction procedures. Furthermore, within the limits of the sample, the catalogues can be compared among each other. Out of the 50 stars included in the astrographical program, $20 \%$ are not in the HIPPARCOS Input Catalogue and $26 \%$ are absent from any optical catalogues used for the plate reductions. A total of 26 sources are VLA astrometric program stars and 10 of them are selected for the planned link of the HIPPARCOS and VLBI systems (Lestrade et al. 1994). Provisional proper motions for the non-HIPPARCOS stars V826 Tau, GG Tau/N, HP Tau/G2 and for the HIPPARCOS star LS 3299 are given.

All the HIPPARCOS stars in the plates have been measured. This should provide a larger scope for analysis of new reductions and also allow us to place the nonHIPPARCOS radio stars in the catalogue system. The 
program is ongoing and we plan to present other results in the near future.

\section{Data and reduction}

The range of declinations extends basically from $+40^{\circ}$ to $-30^{\circ}$. There are just two stars further south: AB Dor at $\delta=-65^{\circ} .5$ and LS3299 at $\delta=-54^{\circ} .3$. Figure 1 shows the sky distribution of the sample. The limit brightness is 14th magnitude. Two fainter stars were observed at the long focus $(16 \mathrm{~m}) 1.60 \mathrm{~m}$ telescope of the Laboratorio Nacional de Astrofisica (LNA) $\left(\phi=-22^{\circ} 32^{\prime} ; \lambda=3^{\mathrm{h}} 2^{\mathrm{m}}\right)$ with a CCD camera, in direct mode, and reduced through a mosaic technique.

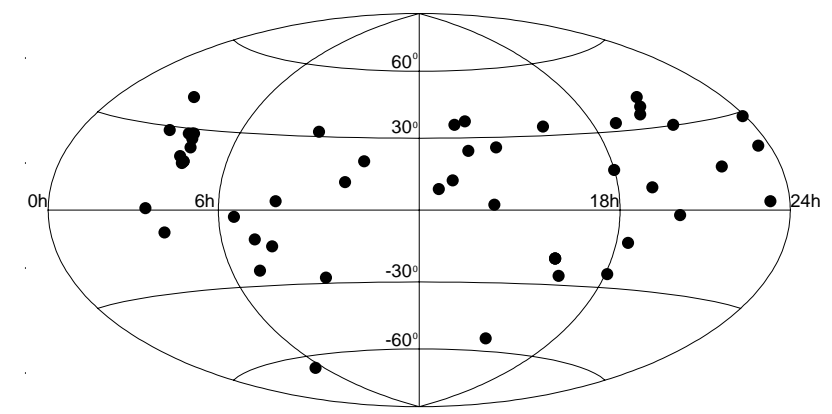

Fig. 1. Sky distribution of the observed radio stars

The main criterion for the selection of stars was the existence of a previously published radio astrometric position. Most of them are RS CVn type, for which a good coincidence of centroids and stable radio emission can be expected (Argue et al. 1984). Detailed astrophysical data have been compiled for each programme star (type, emission, etc.). This information will not be presented here; it is beyond the scope of this paper and will be published elsewhere.

The plates used were Kodak IIaO type, not hypersensitized. Each plate covers a $4^{\circ} \times 4^{\circ}$ region of the sky. Two exposures per plate were taken, with different durations to provide better overall imaging of catalogue stars and radio stars. All the plates were digitized with a PDS 1010A microdensitometer. The plates were scanned in the direct and reverse directions, to account for possible systematic errors of the $\mathrm{x}$ and $\mathrm{y}$ glass encoders, if any. Three successive scans were made for the radio stars, to minimize accidental errors. We restricted the measurements of the stars of the denser catalogues PPM and ACRS to the $3^{\circ}$ $\times 3^{\circ}$ cores of the plate, to avoid excessive scanning time. The $(x, y)$ image centres were determined through two dimension elliptical Gaussian fits to the matrix of pixels of the images. The matrices were previously smoothed with a two dimensional, three channel binomial filter. The outer, noisier pixels were trimmed if located beyond an elliptical area defined by the set of adjusted Gaussian param- eters, in an iterative process. Also, saturated pixels were removed from the fits. More than $95 \%$ of the stars have $(x, y)$ errors smaller than 0. . 05 , derived from the Gaussian adjustments.

At the end, four sets of measurements were obtained per plate ( 2 exposures $\times 2$ directions of measurement), with three $(x, y)$ radio star coordinates for each set. Each set of measurements was reduced independently. A 2nd degree polynomial model plus a 3rd degree radial distortion term was used to relate the measured and standard coordinates of the catalog reference stars (Vieira et al. 1992). Those stars for which the Gaussian adjustment errors exceeded $0 . \prime 08$ were eliminated. A few stars were also eliminated, because they presented spurious "observed minus calculated" residuals (i.e., greater than 0.6 ) in the 6 constant adjustment between the $(x, y)$ direct and reverse measured coordinates of the same star images. That probably happened due to accidental scanning errors of the PDS. For each of the four sets of $(x, y)$ measurements, an independent reduction was performed for each of the catalogues used. The reference stars with greatest "observed minus calculated" residuals were eliminated in a step process, till the radio star equatorial coordinates varied less than $0{ }^{\prime \prime} 01$, which is approximately the average of the errors of the catalogues used, divided by the square root of the average number of catalogue stars. Therefore, each radio star had 12 positions: $3 \times 4$ reference catalogues. The final right ascensions and declinations of the radio stars, in the catalogue system, were the simple arithmetic mean of these 12 positions.

Every HIPPARCOS Input Catalogue star has been measured, so that the re-reduction of plates with the final version of the satellite catalogue will be straightforward. As a by-product, plate positions for each catalogue were derived for all the FK5 and FK5 Extension stars on the plates.

We checked the reduction as a whole by comparing the results for the FK5 and FK5 Extension stars in the plates. The conditions in which the FK5 and FK5Ext stars are found in the plates differ in two ways from the conditions for the radio stars. The FK5 and FK5Ext stars are scattered randomly on the plates and the exposure times were not suitable for their magnitudes. They were generally overexposed and placed far from the plate center. Such conditions are obviously much worse than those for the radio stars.

There are 177 FK5 and FK5Ext stars on the whole batch of plates. The overall results are listed in Table 1. They indicate that all but the PPM averages for the difference "plate minus FK5" are at least of the same order as the FK5 system positional errors. This indicates that the measurement of the plates and their reduction does not introduce a bias in the observed positions as a whole, for the catalogues used. The catalogues themselves appear to be well aligned to the FK5 system. The tendency for 
the PPM, which is also observed for the High Precision Subset (HPS), is found later, when the radio star results are examined.

Table 1. Average plate minus FK5 system results, for the different catalogues used in the reduction

\begin{tabular}{lrrrrrr} 
Reference & \multicolumn{2}{c}{ Right } & \multicolumn{2}{c}{ Ascension } & \multicolumn{3}{c}{ Declination } \\
Catalogue & $\Delta \alpha \cos \delta$ & Error & $\sigma$ & $\Delta \delta$ & Error & $\sigma$ \\
\hline & $(")$ & $(")$ & $(")$ & $(")$ & $(")$ & $(")$ \\
\hline CAMC & 0.01 & 0.04 & 0.38 & 0.03 & 0.04 & 0.36 \\
IRS & -0.03 & 0.03 & 0.33 & 0.01 & 0.04 & 0.44 \\
ACRS & -0.04 & 0.04 & 0.44 & -0.04 & 0.04 & 0.40 \\
PPM & -0.09 & 0.04 & 0.44 & -0.03 & 0.04 & 0.43 \\
HPS & -0.07 & 0.04 & 0.40 & -0.07 & 0.04 & 0.38 \\
\hline
\end{tabular}

However, the scatter of the measured positions is found to be larger than the combined errors of the FK5 system and that of any of the catalogues used. Figure 2 illustrates the effect. From the standard deviation between the results from different catalogues, it is shown that the measuring error becomes larger at the outer regions of the plate. Moreover, the error is larger for the FK5 stars than for the FK5Ext stars, from the center of the plate outwards. This suggests an effect due to both the magnitude and the distance from the center of the star in the plate. After some empirical investigations, such an effect was finally modeled through a least squares routine and a corrective term was adjusted to the positional offsets. It is given by the relations:

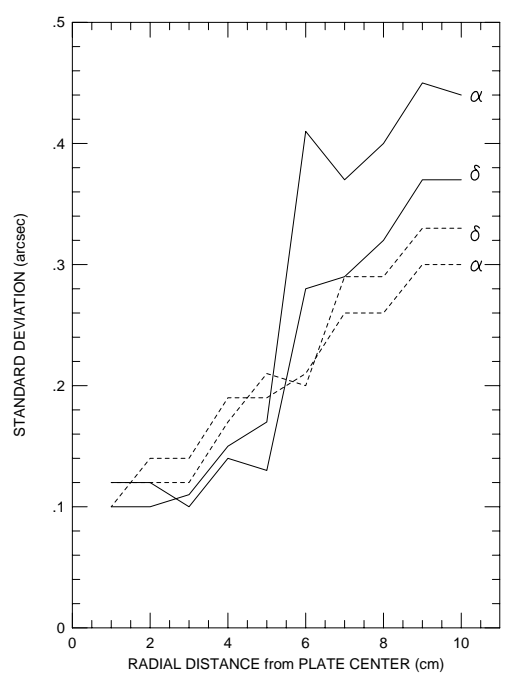

Fig. 2. The increasing scatter of the "plate minus FK5 system" equatorial coordinates offsets, as referred to at least four catalogues, as a function of the distance from the plate centre. The continuous lines refer to FK5 stars and the dotted lines to FK5Ext stars
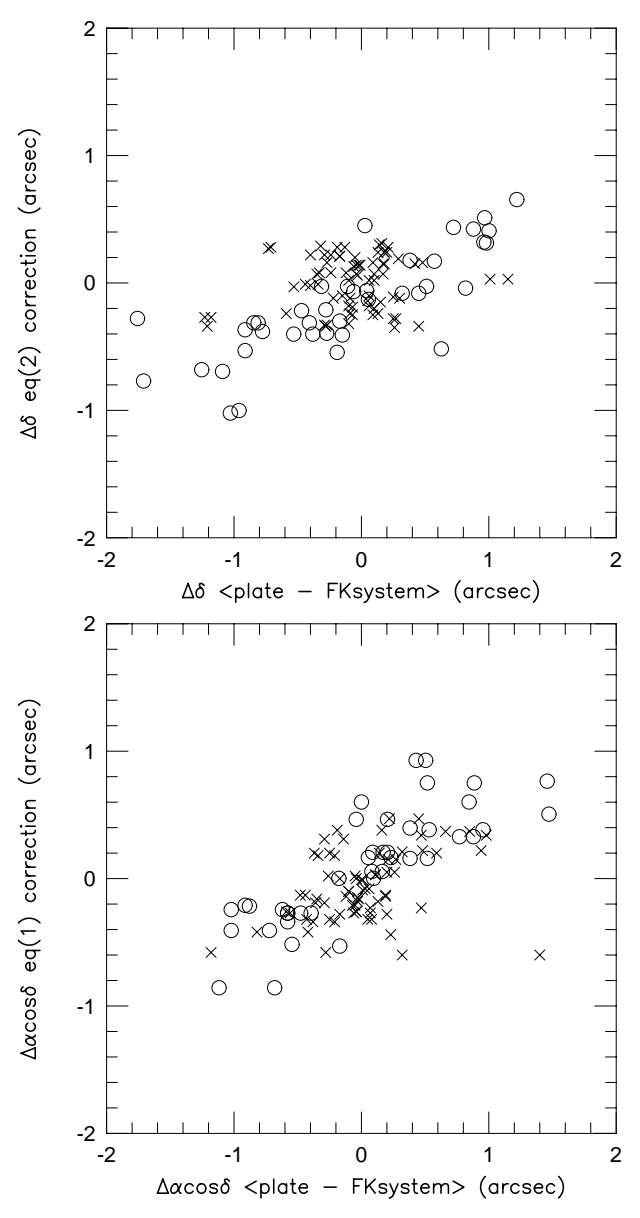

Fig. 3. For right ascension and declination respectively, the correlation between the average "plate minus FK system" offsets and the radial and magnitude correction defined by the relations (1) and (2). Circles refer to FK5 stars and the $\times$ sign to FK5Ext stars

$$
\begin{aligned}
& \Delta \alpha \cos \delta=[A \times R \times \cos (\Theta+B)] / M \\
& \Delta \delta \quad=[A \times R \times \sin (\Theta+B)] / M
\end{aligned}
$$

where $\Delta \alpha \cos \delta$ and $\Delta \delta$ are the right ascension and declination offsets. $A$ is the scaling factor; $R$ the radial distance from the centre in decimeters; $\Theta$ the orientation angle (in radians) on the plate, measured in the direct sense from the direction of increasing right ascensions; $B$ an angle of correction; $M$ the magnitude of the star. The adjustment produced $A=3.715$ and $B=-0.086$ rad.

The use of the correction so defined, halved the standard deviation of the "plate minus FK5" differences. Considering the FK5 error 0'.04 for 1990, it implies a value near 0 '.20 for the standard deviation of scattered bright stars measured in the plates. Figures $3 \mathrm{a}$ and $3 \mathrm{~b}$ show that the corrections given by relations (1) and (2) correlate very well with the offsets between plate results and the FK5 system coordinates. The plate results are given as 
Table 2. Positions obtained astrographically and their comparison against major optical catalogues. The columns in the table present, (1): Name of the radio star; (2): Visual magnitude; (3): Spectral type; (4): Epoch of the astrographic observation; (5): to (7) Right ascension, at epoch, referred to CAMC\#4 catalogue; (8) Right ascension standard error; (9) to (11): Declination, at epoch, referred to CAMC\#4 catalogue; (12): Declination standard error; (13) and (14) respectively, the right ascension and declination differences between the positions obtained from the plate reductions (referred to the CAMC\#4 catalogue) and the positions given in the CAMC\#4 catalogue; (15) and (16) respectively, the right ascension and declination differences between the plate positions as obtained referred to the CAMC\#4 and ACRS catalogues; (17) and (18) analogous to the previous, for the PPM catalogue; (19) and (20) analogous to the previous, for the High Precision Subset of the PPM catalogue; (21) and (22) analogous to the previous, for the IRS catalogue

\begin{tabular}{|c|c|c|c|c|c|c|c|c|c|c|c|c|c|c|c|c|c|c|c|c|c|}
\hline \multirow[t]{2}{*}{ Name } & \multirow[t]{2}{*}{ Mag } & \multirow[t]{2}{*}{$s p$} & \multicolumn{2}{|l|}{$E_{\mathrm{P}}$} & \multicolumn{2}{|c|}{$\alpha(\mathrm{J} 2000)$} & $E \mathbf{r}_{\alpha \boldsymbol{\alpha}}$ & \multicolumn{3}{|c|}{$8(J 2000)$} & \multirow{2}{*}{$\begin{array}{l}\mathbf{E}_{\Sigma_{\delta}} \\
\end{array}$} & \multicolumn{2}{|c|}{$\begin{array}{l}\mathrm{CAMC}_{\mathrm{pl}}-\mathrm{CAMC}_{\mathrm{cat}} \\
\Delta \alpha \cos \delta \quad \Delta 8\end{array}$} & \multicolumn{2}{|c|}{$\begin{array}{l}\text { CAMC }_{\mathrm{PU}}-\mathrm{ACRS}_{\mathrm{k}} \mathrm{L} \\
\Delta \alpha \mathrm{OSO} \delta\end{array}$} & \multicolumn{2}{|c|}{$\underset{\Delta \alpha \operatorname{CAMC} \mathrm{CS}_{\overline{1}}-\mathrm{PPM}}{\Delta \overline{\mathrm{O}}}$} & \multicolumn{2}{|c|}{$\begin{array}{l}\mathrm{CAMC}_{\mathrm{OL}}-\mathrm{HPS}_{\mathrm{ZI}} \\
\Delta Q \cos \delta\end{array}$} & $\begin{array}{c}\mathrm{CAMC}_{\mathrm{B}}- \\
\Delta \alpha \cos \delta\end{array}$ & 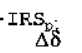 \\
\hline & & & $1900+$ & $\mathrm{b}$ & $\mathrm{m}$ & $\sec$ & $\sec$ & \% & ' & " & & $"$ & 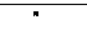 & $\cdot$ & " & " & $\pi$ & $\pi$ & " & " & $"$ \\
\hline$g \circ 1$ & 2.60 & B9 & .65 & 3 & 8 & 10.135 & 005 & +40 & 57 & 20.67 & 0.05 & & & 0.15 & 0.16 & 0.50 & $0 . \overline{11}$ & 0.18 & 0 . & 0.20 & 0 \\
\hline Ari & 5. 60 & G5 & 92.65 & 3 & 26 & & & +28 & 42 & 55.00 & 0.09 & .21 & 09 & -0. & -0. & 0.0 & 0.14 & & & & \\
\hline 1 & 5.71 & G5 & 92.65 & 3 & 36 & 47. & .005 & & 35 & 17.08 & 0.05 & of & & 0.0 & -0. & 0.32 & 0.12 & & & & -0.19 \\
\hline EI Eri & 7.10 & $\mathrm{G} 2$ & 92.73 & 4 & 9 & 40.880 & 0.021 & -7 & 53 & 35.14 & 0.03 & 0.0 & 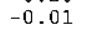 & 0.28 & -0.2 & 0.08 & -0.16 & & & -0 . & -0.06 \\
\hline 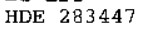 & 10.59 & K3 & 93.87 & 4 & 14 & 12.925 & 0.004 & +28 & 12 & 12.41 & 0.03 & & & & 0.1 & -0.05 & -0.03 & -0. & 0.05 & -0. & -0.08 \\
\hline$\forall 41$ & 11.00 & $\mathrm{~K} 7$ & 93.80 & 4 & 18 & 31.127 & 0.006 & +28 & 27 & 16.16 & 0.11 & & & 0.07 & 0.05 & -0.05 & -0.06 & & & -0.09 & 0.30 \\
\hline $\mathrm{HI}$ & 9.00 & G5 & 93.87 & 4 & 21 & 58.817 & 0.020 & +28 & $1 \mathrm{~B}$ & 6.39 & 0.09 & & & -0.39 & -0.51 & -0.32 & -0.23 & -0.34 & 0. & & 0.33 \\
\hline & 10.00 & F8 & 92.6 .6 & 4 & 21 & 59.436 & 0.007 & +19 & 32 & 6.36 & 0.12 & 0.19 & -0.2 & 0.12 & 0.10 & 0.02 & 0.18 & 0. & 1 & -0.05 & 0.32 \\
\hline & 12.00 & MO & 92.73 & 4 & 27 & 4.697 & 0.008 & +26 & 5 & 15.83 & 0.14 & & & -0. & -0.25 & -0.09 & -0.30 & & & & -0.27 \\
\hline 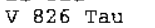 & 12,11 & K 7 & 93.87 & 4 & 32 & & 0.009 & +18 & 1 & 38.72 & 0.12 & & & 0. & 0. & 0.42 & 0.18 & 0. & 0. & -0. & 0.07 \\
\hline N & 13.70 & $\mathrm{~K} 5$ & 92.73 & 4 & 32 & & 0.005 & +17 & 31 & 40.68 & 0.0 & & & 0. & -0. & 0.32 & 0.21 & & & & -0.24 \\
\hline $\mathrm{HD} 2$ & 6.25 & B9 & 93.87 & 4 & 33 & 32 & 0.005 & +18 & 1 & 0.41 & 0. & & & & -0 & & 0. & & & & 0.01 \\
\hline HF Tau/G2 & 13.40 & $\mathrm{~K} 3$ & 92.66 & 4 & 35 & 54.3 & 0.010 & +22 & 54 & 13. & 0.13 & & & -0 & -0 . & -0.17 & -0.42 & & & -0. & \\
\hline AB Dor & 6.70 & G8 & 92. & 5 & 28 & 44.8 & 0.0 & -65 & 26 & & 0.06 & & & 0 & 0. & & -0.08 & & & -0 . & \\
\hline 614 & 11.40 & $\mathrm{M} 7$ & 93.30 & 6 & 29 & 23.1 & 0.009 & -2 & 48 & 46.02 & 0.10 & & & 0 & -0 . & 0 . & -0 . & & & 0. & -0.16 \\
\hline & & B5 & 92. & 6 & 54 & 13. & 0 & -23 & 55 & 42.05 & 0.08 & 3 & 0.18 & 0. & 0. & 0. & 0.17 & & & & 0.34 \\
\hline $\mathrm{CN}$ & 10.00 & $\mathrm{~B} 8$ & 92.89 & 7 & 3 & 43. & 0.007 & -11 & 33 & 5.96 & 0.08 & & & & 0.04 & -0. & 0.09 & & & & 0.13 \\
\hline KQ Pup & 5.40 & $\mathrm{B2}$ & 92.89 & 7 & 33 & 47. & 0.0 & -14 & 31 & 26.09 & 0.08 & 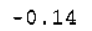 & 006 & -0 & 0.02 & 0. & 0.11 & & & -0 & 0.09 \\
\hline Yiz CMi & 10.80 & M4 & 93.31 & 7 & 44 & 40. & 0.0 & +3 & 33 & 11.80 & 0.31 & & & 0 & -0 . & 0 . & -0.09 & & 0.13 & & \\
\hline RZ Cnc & 9.35 & $\mathrm{~K} 1$ & 93.30 & 8 & 39 & & 0.0 & +31 & 47 & 44.66 & 0.1 & & & 0 & 0. & 0 . & 0.36 & & 0 & & \\
\hline TY FYX & 7.20 & 62 & 93.30 & 8 & 59 & 42. & 0. & -27 & 48 & & 0. & 0.13 & & & & & 0. & & & & 3 \\
\hline R Leo & 7.80 & M8 & 94. & 9 & 47 & 33. & 0 & +11 & 25 & 0 & 0.08 & 0.3 & & -0 & & 0. & -0.11 & 0.00 & 0.06 & -0 & 0.01 \\
\hline $\mathrm{AD}$ Leo C & 9.60 & Ma & 93.30 & 10 & 19 & 36.4 & 0.0 & +19 & 52 & 12.24 & 0.1 & & & & -0. & & 0.01 & & 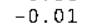 & & {$[0$} \\
\hline 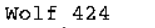 & & M5 & & 12 & 33 & & & +9 & 1 & & & & & & & & 0. & -0 . & & & 0.37 \\
\hline DT Vir & 34 & $\mathrm{Mz}$ & 9 & 13 & 0 & & 4 & +12 & 22 & 32.62 & 0. & & & 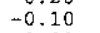 & -0 . & & -0.14 & & 0. & & -0.24 \\
\hline RS CVn & 8 & F5 & & 13 & 10 & & & +35 & 56 & & & & & & & & & & & & 0.19 \\
\hline FK Com & 8 & Go & 9 & 13 & 30 & & & +24 & 13 & 57. & 0. & & & & 0 . & & 0. & & & & 0.27 \\
\hline $\mathrm{cvn}$ & 1 & $\mathrm{~F} 2$ & & 13 & 34 & & & +37 & 10 & & 0. & & & & -0. & & -0. & & & & \\
\hline CU Vir A & 4.92 & B9 & 93.30 & 14 & 12 & 15 & 0.0 & +2 & 24 & & 0. & 0.21 & & & 0. & -0 . & 0 . & & & & 56 \\
\hline RX BOO & 8.00 & M7 & 93.30 & 14 & 24 & 11. & 0.0 & +25 & 42 & & 0. & 0.0 & & & -0. & & 0. & 0. & 0 . & & \\
\hline LS 3299 & & B5 & & 14 & 59 & 53 & & -54 & 18 & 7.66 & 0.1 & & & 0. & 0.04 & -0.09 & -0.07 & & & 0. & -0.80 \\
\hline oph 1 & & $\mathrm{~K} 2$ & 93. & 16 & 11 & & & -19 & 4 & & 0.1 & & & & & & -0.07 & & & & \\
\hline & 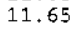 & & & 16 & 11 & & & -19 & 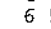 & & & & & & -0 & & -0. & & & & \\
\hline oph 3 & 12.30 & $\mathrm{KO}$ & 93.56 & 16 & 12 & 40 & & -18 & 59 & & 0. & & & & & & -0. & & & & \\
\hline$\sigma c r b$ & & F8 & & 16 & 14 & & & +33 & 51 & & & & & & -0 & & & -0.09 & 0 & & \\
\hline a. sco A & 1.35 & $\mathrm{~B} 2$ & 92.66 & 16 & 29 & 24 & & -26 & 25 & & 0. & & & & & -0. & -0. & & & & 0.14 \\
\hline $\mathrm{z}$ Her & 7.30 & F7 & 92.65 & 17 & 58 & 7.8 & 0. & & 8 & & 0. & & & & & & 0. & 0.2 & 0.7. & & \\
\hline $95 g r$ & 4.80 & 05 & 92.73 & 18 & 3 & 52.444 & 0.0 & -24 & 21 & & 0.0 & -0.0 & 0. & 0. & -0.07 & 0. & 0.18 & & & -0. & -0.19 \\
\hline FR Set & 10.00 & M3 & 92.65 & 19 & 23 & 22.8 & 0.0 & -12 & 40 & & & 0.1 & 0 & & -0 . & -0 & -0 . & & & & \\
\hline$\beta$ Lyr & & $\mathrm{B} B$ & 92.65 & 18 & 50 & & 0.0 & +33 & 21 & 45.61 & 0.1 & & & & -0. & & -0 & & -0 . & & \\
\hline R Agl $A$ & 8.00 & M & 92.73 & 19 & $c$ & & 0.0 & +8 & 13 & & 0.0 & & & & & & & & & & \\
\hline $\mathrm{RR}$ Aql & 1.2 .1 & M6 & & 19 & 57 & & & -1 & 53 & & & & & & & & -0 . & & -0. & & \\
\hline $\mathrm{CYg}-\mathrm{XI}$ & & Bo & & 19 & 58 & & & +35 & 12 & & & -0. & & -0 . & & & 0 & -0 & & & 23 \\
\hline $\mathrm{P}$ CYO & & 89 & & 20 & 17 & & & +38 & 1 & & & & & & & & o. & & & & 03 \\
\hline $\mathrm{CYg}$ OBZ & 9.30 & 06 & & 20 & 32 & & & +41 & $1 \overline{8}$ & & & & & & & & -0 . & & & & 0.33 \\
\hline UX CYg & 10.20 & M4 & 94.69 & 20 & 55 & & & +30 & 24 & & & & & -0. & $=0$. & & 0. & & -0. & & \\
\hline HN Feg & 5 & Go & 92 & $2 I$ & 44 & & & +14 & 46 & & & & & & & & 0. & & 0 . & & 0.63 \\
\hline SZ Psc & 7.18 & K1 & 92.65 & 23 & 13 & & & +2 & 40 & & 0. & & & & & & 0 . & & & & \\
\hline EQ Peg A & 10.2 & M4 & & 23 & 31 & & & +19 & 56 & & & & & & -0. & & -0. & & & & \\
\hline II Feg & 7.18 & $\mathrm{~K} 1$ & & 23 & 5 & 732 & & +28 & 38 & & 0.1 & 0.02 & & & 0. & 0.36 & 0.42 & & & & \\
\hline
\end{tabular}

averages for every star that was measured relative to at least four of the reference catalogues. Although the nominal errors of the FK5Ext are larger, the magnitude effect makes the correlation more evident for the FK5 stars.

The values of the corrections drop rapidly for radial distances smaller than $2 \mathrm{~cm}$ and for magnitudes fainter than 6 . Thus, most of the radio stars have been virtually unaffected by the effect. The correction was defined by FK5 and FK5Ext stars and it was applied only for the radio stars brighter than eighth magnitude. Its value is larger than 0 .'05 for only four stars. UX Ari has corrections of -0 .'10 and -0 .'08 applied to the measured right ascension and declination, respectively. AB Dor has correction of +0 .'08 in right ascension and +0 .' 07 in declination. These two stars have been affected by measurements out of the plate center. $\alpha$ Sco A, the brightest star of our sample, has a correction of -0 .'09 in declination.

\section{Results}

The plate reductions have been made relative to the following optical catalogues: the CAMC \#4, (declinations above $-40^{\circ}$, with an average of 15 stars per plate), the ACRS (average of 44 stars per plate), the PPM (average of 55 stars per plate) and the IRS. For the latter, the star density is too small to enable a complete solution for the plate constants. A previous reduction with the ACRS was then done to solve for the plate constants, after which the 
IRS stars were used to solve for the position. The same method was used for the HPS (average of 15 stars per plate) and for a second solution with the CAMC. The comparison between both methods for the CAMC showed no introduction of systematic deviations, but the accuracy was degraded by about $0 . \prime 05$. The average rms errors for CAMC plate reductions were 0 "'20 and 0 "' 17 for right ascension and declination, respectively.

Table 2 displays the results. The positions (in Cols. 5 to 7 and 9 to 11) and standard errors (in Cols. 8 and 12) are given in the CAMC system, except for AB Dor $(\delta=$ $\left.-65^{\circ} 5\right)$ and LS3299 $(\delta=-54.3)$, which are given in a hybrid system combining the ACRS and the PPM. The plate positions for the other reference catalogues are presented as differences from the CAMC plate positions (Cols. 15 to 22). The IRS reference positions are obtained through the ACRS reduction explained above, while the HPS positions have no correction applied. The radius/magnitude correction discussed in Sect. 2 is applied in the presented values.

In Table 2 we present the differences between the equatorial coordinates obtained in the reduction and the catalogue coordinates, for the radio stars present in the CAMC. The corresponding differences for each catalogue are plotted in Figs. 4a to 4d. The average differences between plate solution and the catalogues are in Table 3. The best match occurs with the CAMC, probably due to the high quality of the coordinates and proximity of the catalogue mean epoch to the average epoch of observation of the plates. This, together with the higher star density relative to the IRS, made us choose to present the results in the CAMC system. The tendency for a systematic right ascension displacement found between the FK5 and the PPM systems is found again between the CAMC and the PPM, in the same sense. For the HPS the right ascension displacement vanishes in this case.

Table 3. Average plate minus catalogue results

\begin{tabular}{lccrr} 
Reference & \multicolumn{2}{c}{ Right Ascension } & \multicolumn{2}{c}{ Declination } \\
Catalogue & $\Delta \alpha \cos \delta$ & $\sigma$ & \multicolumn{1}{c}{$\Delta \delta$} & $\sigma$ \\
\hline & $(")$ & $(")$ & $(")$ & $(")$ \\
\hline CAMC & -0.02 & 0.21 & -0.02 & 0.17 \\
IRS & 0.01 & 0.27 & 0.02 & 0.33 \\
ACRS & -0.07 & 0.26 & 0.02 & 0.25 \\
PPM & -0.13 & 0.31 & -0.06 & 0.19 \\
HPS & 0.01 & 0.25 & -0.08 & 0.18 \\
\hline
\end{tabular}

As the main criterion for including stars in the program is not tied to any specific survey, the resulting distribution of stars (see Fig. 1) is close to isotropic in a wide equatorial belt. It is not, however dense enough to allow for a systematic study of zonal errors of the catalogues, unless direct measures of the extragalactic or the HIPPARCOS systems are employed, since these are much more precise and very rigid. Nevertheless, the analysis of the dependencies on right ascension and declination might offer some indications, as well as corroborate the results obtained.

In Figs. 5a to 5d we plot the smoothed curves of the $\Delta \alpha \cos \delta$ and $\Delta \delta$ differences of the plate results as referred to the CAMC and to the other catalogues. For comparison, the average values of the corresponding offsets, in the region of the plates, are also plotted. The plate differences and the average catalogue offsets are in overall agreement. The maximum scatter is of the order of $00^{\prime \prime} 30$, which is consistent with the combined error of the catalogues.

The smoothed lines corresponding to the different plate results are very much alike, indicating that the ACRS and PPM systems are well aligned. It is more difficult to discuss the IRS results, since for this catalogue a previous plate reduction was made with ACRS stars. We believe the IRS results to be reliable as a whole, as shown by the averages in Table 3. In the same table, however, the scatter of the IRS results appears to be larger than expected from the catalogue nominal errors.

Local features are present in all figures. More noticeably, a positive slope of the differences in right ascension, between 12 and 20 hours. On the $\Delta \delta_{\delta}$ plot there is a large bulge for negative declinations. For declinations larger than $+20^{\circ}$, there is a divergence of the discrete symbols (i.e, the average catalogue offsets) and the smoothed lines (the plate offsets) which is probably of an observational nature. At latitude $-23^{\circ}$, the stars observed in that zone are more affected by refraction effects.

From Table 3, the average offsets between the catalogue positions and those obtained through the plate reductions was smaller than $0^{\prime \prime} 03$ for both the equatorial coordinates when the CAMC and the IRS were used. The average CAMC right ascension and declination rms errors of the radio stars are 0.20 and $0^{\prime \prime} 17$ respectively. These values are close to the corresponding standard deviations of Table 3. Considering the average number of 15 CAMC stars per plate and the $0 . \prime 05$ error of the $(x, y)$ Gaussian adjustments, the average rms errors found lead to an $0{ }^{\prime \prime} 07$ plate error for both right ascension and declination of a star. Bright radio stars will probably display higher plate errors due to measurement degradation and to the radial/magnitude effect. In this case, a plate error of 0 "' 10 up to 0 '. 20 might be expected, based in the analysis of the FK5 plate positions in the preceding section.

Two stars, Lk H $\alpha 101$ and HBC 362, are too faint to be conveniently observed with the Zeiss astrograph in Campinas. They were observed at the $1.60 \mathrm{~m}$ telescope of the LNA and had their positions obtained through a mosaic of CCD frames. The method of reduction is fully described elsewhere. The results for Lk $\mathrm{H} \alpha 101$ and HBC 362 are in Table 4.

Nine stars in our sample have no proper motion indicated in the literature. We were able to use previous 

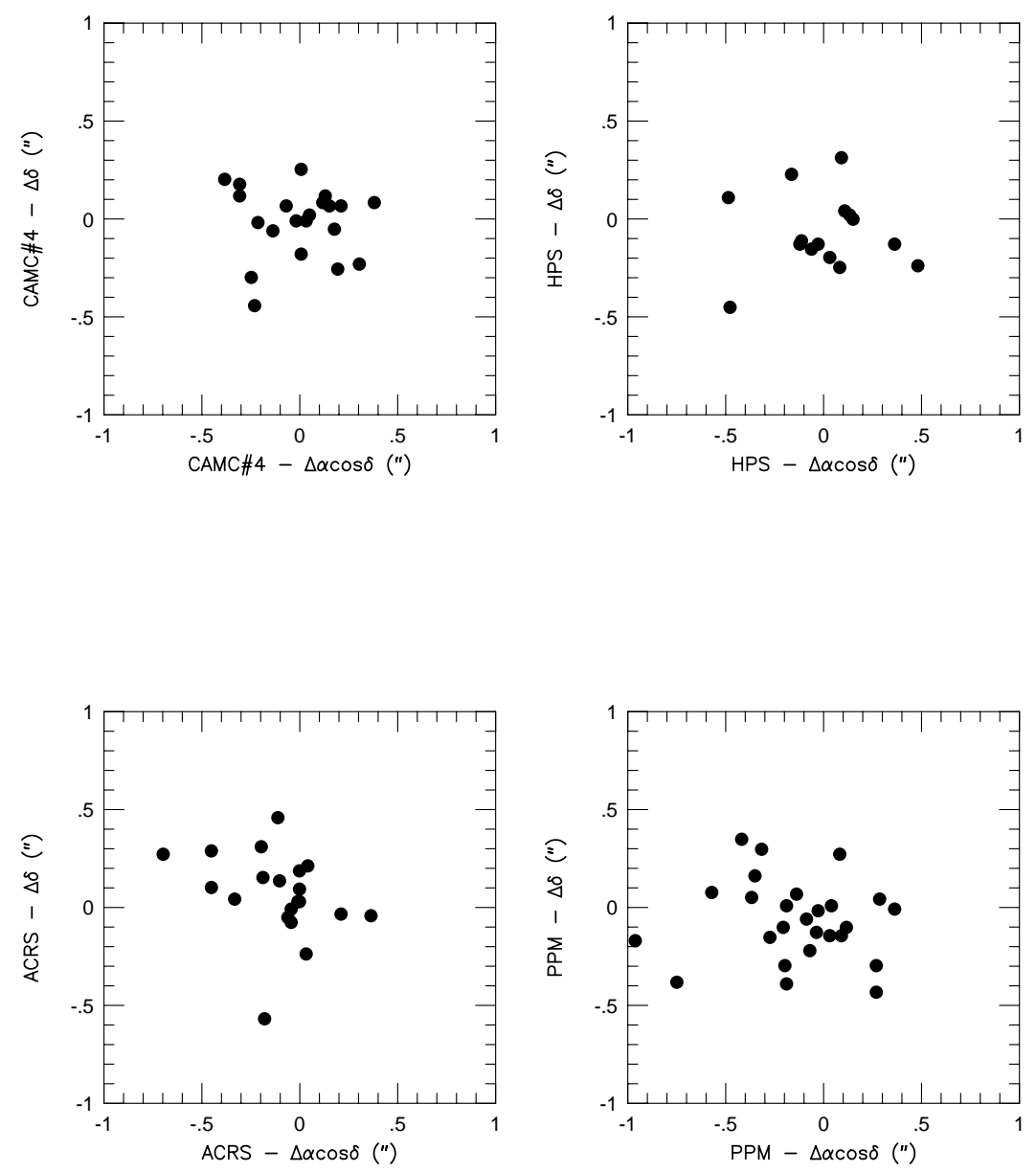

Fig. 4. The differences in equatorial coordinates for every radio star belonging to one of the catalogues used for the plate reduction. The differences are in the sense "catalogue minus plate"

Table 4. Positions obtained by mosaicing of CCD images and their comparison against major optical catalogues. All radio star positions referred to the CAMC\#4 catalogue through CCD reductions with Guide Star Catalogue stars corrected to the CAMC\#4. Layout as in Table 2

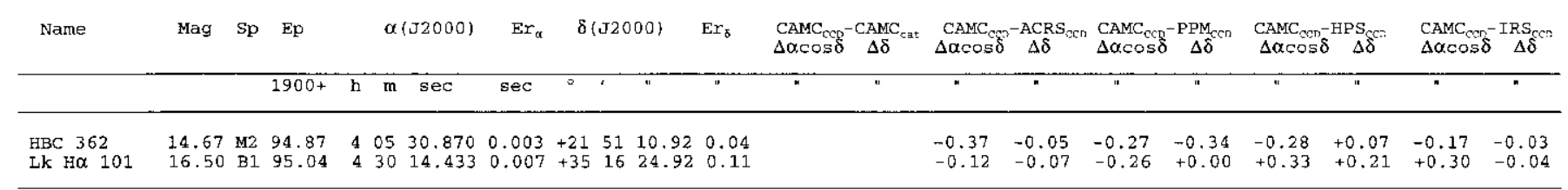

position determinations for five of those to calculate provisional proper motions. The positions were obtained from the literature referred to in Wendker (1993) and found in the SIMBAD database.

For these stars we used both radio and optical positions, the exception being GG Tau/N, for which there is evidence that the radio and optical emissions are not associated with the same object. Only one of the stars belongs to the HIPPARCOS catalogue, LS3299, a super- priority radio star. Its quoted radio position (Wright et al. 1974), however, has a very large $\left(\sim 10^{\prime \prime}\right)$ uncertainty. The collected optical positions indicate that the radio position appears to be displaced about $0.5 \mathrm{~s}$ eastward and $7^{\prime \prime}$ northward. Due to its large uncertainty, the radio position contribution for the provisional proper motion is negligible. The optical position of V826 Tau, quoted by Kutner et al. (1986) is also discrepant ( $\sim 2^{\prime \prime}$ southward) and, due to its large uncertainty, negligible to the calculated proper 

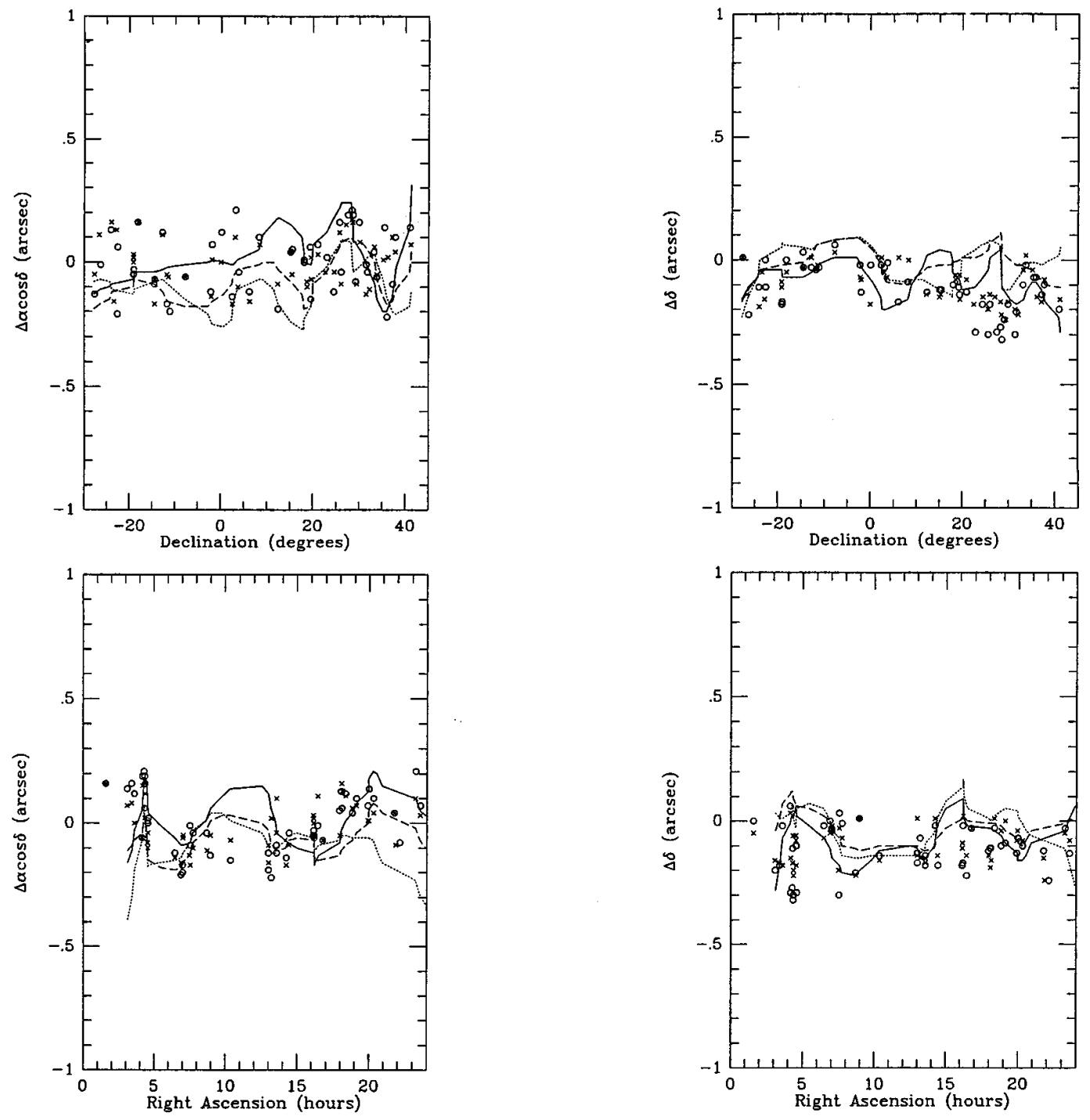

Fig. 5. The smoothed lines represent the differences between the plate results, as obtained from the reference catalogues. The continuous line shows PPM minus CAMC; the dashed line ACRS minus CAMC; the dotted line IRS (with a previous ACRS reduction) minus CAMC; $\times$ represent the PPM minus CAMC and open circles the ACRS minus CAMC systematic differences between the catalogues themselves in the plate region

motion. Finally, the mosaic position for Lk H $\alpha 101$ in Table 4 disagrees with the other collected positions by about $00^{\prime \prime} 5$ in declination and was discarded for the calculation of the proper motion. Positions for these stars have been found in White et al. (1992), Herbig \& Rao (1972), for GG Tau/N; Cohen \& Bieging (1986), for HP Tau/G2; Herring et al. (1986), Le Bertre et al. (1989) and Costa \& Loyola (1990), for LS3299; Spencer \& Schwartz (1974) and Clements \& Argyle (1984), for Lk H $\alpha 101$.

The proper motions are presented in the FK5 system. Corrections were made to account for the systematic differences between the FK4 and FK5 systems, including the presence of aberrational elliptic terms. Regarding our purpose, we did not attempt to correct for zonal errors between catalogue systems other than these referred to above. The results are given in Table 5 .

\section{Conclusions}

We observed optically fifty stars, for all of which there is a radio astrometric determination of position.

FK5 and FK5Ext stars were used to check the procedures. Although they are found scattered over the plates, contrary to the radio stars which are always the central object, the position offsets obtained for the FK5 and FK5Ext stars validated the methods used and enabled us to establish a radial/magnitude correction for the reduced positions of the radio stars. The average offset between the FK5 catalogue positions and the positions 
Table 5. Provisional proper motions. $N$ indicates the number of used positions and $\Delta E_{\mathrm{p}}$ the largest difference of epochs

\begin{tabular}{lrrrrrr} 
Star Name & $\mu_{\alpha}$ & Error & $\mu_{\delta}$ & Error & $N$ & $\Delta E_{\mathrm{p}}$ \\
& $\mathrm{s} / \mathrm{yr}$ & $\mathrm{s} / \mathrm{yr}$ & " $/ \mathrm{yr}$ & " $/ \mathrm{yr}$ & & $\mathrm{yr}$ \\
\hline V826 Tau & 0.001 & 0.001 & -0.05 & 0.01 & 4 & 10.9 \\
GG Tau/N & 0.000 & 0.004 & -0.02 & 0.04 & 2 & 22.7 \\
HP Tau/G2 & -0.002 & 0.001 & -0.06 & 0.01 & 3 & 10.6 \\
LS3299 & 0.002 & 0.001 & -0.01 & 0.01 & 5 & 35.6 \\
Lk H $\alpha$ 101 & 0.001 & 0.001 & 0.00 & 0.02 & 5 & 30.1 \\
\hline
\end{tabular}

obtained through the plate reduction was smaller than 0 .'03 for both the equatorial coordinates when the CAMC and the IRS were used. The Gaussian $(x, y)$ measurement errors are $0 . \prime 05$. The rms plate errors of the CAMC reference stars are $00^{\prime \prime} 20$ and $0{ }^{\prime \prime} 17$ for right ascension and declination. The plate error of an individual star position is estimated as $0{ }^{\prime \prime} 07$. For brighter stars, it may rise from $00^{\prime \prime} 10$ up to $0^{\prime \prime} .20$ due to measurement degradation and to the radial/magnitude effect.

The analysis of the results has shown the CAMC system is better aligned to the FK5 system than any of the other catalogues used and with smaller errors for individual reference stars. The results are thus presented in the CAMC system and the corrections from this to the other catalogues are indicated for each radio star.

For the stars V826 Tau, GG Tau/N, HP Tau/G2, LS3299 and Lk $\mathrm{H} \alpha$ 101, we calculated provisional, raw values for the proper motion, which were not found in the literature, taking the first epoch positions from the SIMBAD database and from Wendker (1993).

The comparison between the catalogues seems to indicate that the ACRS and PPM are well aligned. The PPM zero point of right ascension appears to be displaced some 0 .'05 to the east from the FK5 and CAMC origins. For southern declinations, we found a significant bulge feature in the $\Delta \delta$ differences between the CAMC and the other catalogues. Overall, the differences are not larger than $0 . \prime 3$.

Forty of the stars presented here belong to the HIPPARCOS catalogue. The other 10 stars that do not (mostly due to their faintness) can help to extend the HIPPARCOS reference frame to fainter magnitudes. As soon as the HIPPARCOS results are available, the HIPPARCOS reference stars already measured in the plates will allow us to reduce the 10 non-HIPPARCOS radio stars in the catalogue system.

Acknowledgements. We acknowledge the Las Campanas Observatory for the donation of the plates used in this work. We also acknowledge the use of the facilities of the Laboratório Nacional de Astrofísica LNA/CNPq and of the Observatório Municipal de Campinas (Observatório do Valongo/UFRJ). We are specially grateful to our colleagues Charles Rité and Mar$\cos$ A. Nunes for their constant assistance on all kind of hardware matters. This research was partially supported by F.U.S.B./U.F.R.J. grant No. 3914-4. Three of us (SP, EJ and WO) thank the $\mathrm{CNPq} /$ Brazil for their grants. This research has made use of the SIMBAD database, operated at CDS, Strasbourg, France.

\section{References}

Andrei A.H., Jilinski E.G., Puliaev S.P., 1995, AJ 109, 428

Argue A.N., de Vegt Ch., Elsmore B., et al., 1984, A\&A 130, 191

Le Bertre T., Epchtein N., Gouiffes C., Heydari-Malayeri M., Perrier C., 1989, A\&A 225, 417

Carlsberg Meridian Catalogues No. 4, 1990, Copenhagen University Observatory, Royal Greenwich Observatory and Real Instituto y Observatorio de la Armada en San Fernando

Clements E.D., Argyle R.W., 1984, MNRAS 209, 1

Cohen M., Bieging J.H., 1986, AJ 92, 1396

Corbin T.E., 1991, International Reference Stars Catalog; Washington, U.S. Naval Observatory

Corbin T.E., Urban S.E., 1991, "Astrographic Catalogue Reference Stars; Doc. No. NSSDC/WDC)A)R\&S 91)10, Greenbelt, U.S.A.

Costa E., Loyola P., 1990, A\&AS 83, 235

Herbig G.H., Rao N.K., 1972, ApJ 174, 401

Hering R., Schwerdtfeger H.-M., Walter H.G., 1986, Internal record, Astron. Rechen-Institut, Heidelberg, 16 May 1986

Jones B.F., Cohen M., Wehinger P.A., Gehren T., 1987, AJ 94, 1260

Lestrade J.-F., Jones D.L., Preston R.A., et al., 1994, A\&A (in press)

Niell A.E., Lestrade J.-F., Preston R.A., Mutel R.L., Phillips R.B., 1988, "The impact of $V L B I$ on Astrophysics and Geophysics". In: Reid M.J. and Moran J.M. (eds.) by the IAU

Réquième Y., Mazurier J.M., 1991, A\&AS 89, 311

Röser S., Bastian U., 1993, Bull. Inf. CDS 42, 11

Spencer J.H., Schwartz P.R., 1974, ApJ 188, L105

de Vegt Ch., Gehlich U.K., 1982, A\&A 113, 213

Vieira G.G., Assafin M., Vieira Martins R., 1992, PASP 104 467

Walter H.G., Hering R., Lenhardt H., de Vegt Ch., Florkowski D.R., Johnston K.J., 1993, Proceedings on the 156th Symposium of the IAU on "Developments in Astrometry and their Impact on Astrophysics and Geodynamics", Shangai (China) Sept. 15-19 1992. In: Mueller I.I. and Kolaczeck B. (eds.). Kluwer Academic Publishers, 377

White S.M., Pallavicini R., Kundu M.R., 1992, A\&A 257, 557

Wright A.E., Fourikis N., Purton C.R., Feldman P.A., 1974, Nat 250, 715 